\title{
Dark Energy and Dark Matter
}

\author{
Jeevan Regmi \\ Department of Physics, Prithvi Narayan Campus, Pokhara \\ jsregmi@hotmail.com
}

\begin{abstract}
The new discoveries and evidences in the field of astrophysics have explored new area of discussion each day. It provides an inspiration for the search of new laws and symmetries in nature. One of the interesting issues of the decade is the accelerating universe. Though much is known about universe, still a lot of mysteries are present about it. The new concepts of dark energy and dark matter are being explained to answer the mysterious facts. However it unfolds the rays of hope for solving the various properties and dimensions of space.
\end{abstract}

Keywords: dark energy, dark matter, accelerating universe, space-time curvature, cosmological constant, gravitational lensing.

\section{INTRODUCTION}

It was Albert Einstein first to realize that empty space is not 'nothing'. Space has amazing properties. Many of which are just beginning to be understood. The first property that Einstein discovered is that it is possible for more space to come into existence. And his cosmological constant makes a prediction that empty space can possess its own energy. Theorists still don't have correct explanation for this but they have given it a name as 'dark energy'.

Dark energy makes up approximately $70 \%$ of the universe and appears to be associated with the vacuum in space. It is distributed evenly throughout the universe, not only in space but also in time - in other words, its effect is not diluted as the universe expands. The even distribution means that dark energy does not have any local gravitational effects, but rather a global effect on the universe as a whole. This leads to a repulsive force, which tends to accelerate the expansion of the universe. The rate of expansion and its acceleration can be measured by observations based on the Hubble law. These measurements, together with other scientific data, have confirmed the existence of dark energy and provide an estimate of just how much of this mysterious substance exists.

In order to explain acceleration of universe, we need "dark energy" with large negative pressure to generate a repulsive gravitational force. The evidence comes from studies of the total energy density of the universe and from supernova observations. Precision measurements of the cosmic microwave background (CMB) have shown that the total energy density of the universe is very near the critical density needed to make the universe flat (i.e. the curvature of space-time, defined in General Relativity, goes to zero on large scales). Since energy is equivalent to mass (Special Relativity: $\mathrm{E}=\mathrm{mc}^{2}$ ), this is usually expressed in terms of a critical mass density needed to make the universe flat. Ordinary matter such as stars, dust, and gas account for only $5 \%$ of the necessary mass density. Observations have shown that dark matter cannot account for more than $\sim 25 \%$ of the critical mass density. Both the microwave background and supernova observations suggest that dark energy should make up $\sim 70 \%$ of the critical energy density. When added to the massenergy of matter, the total energy density is consistent with what is needed to make the universe flat.

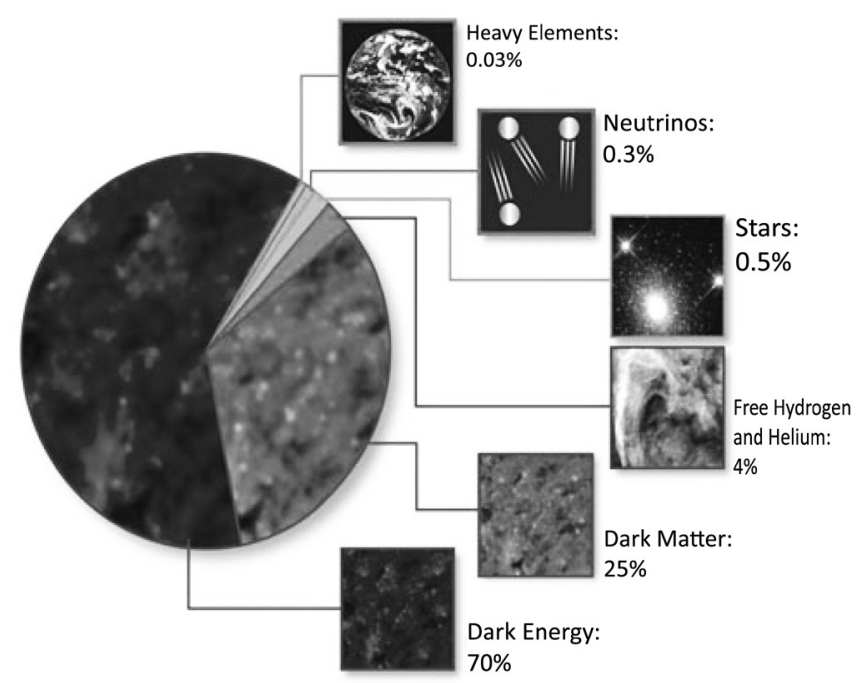

Fig1: An approximate outline of composition of cosmos 
Cosmologists understand almost nothing about dark energy even though it appears to comprise about 70 percent of the universe. They are desperately seeking to uncover its fundamental properties: its strength, its permanence, and any variation with direction. The evolution of the universe is governed by the amount of dark matter and dark energy. The densities of dark matter and dark energy scale differently with cosmic scale as the universe expands. In a universe with a high density of dark matter, the Hubble expansion continues to decelerate due to the gravitation attraction of the dark matter filling the universe, ending in a big crunch. In a universe with a lower critical density of dark matter, the expansion costs. In a universe with dark energy as well as dark matter, the initial deceleration is reversed at late times by the increasing dominance of the dark energy.

If the hypothetical dark energy continues to dominate the universe's energy balance, then the current expansion of space will continue to accelerate, exponentially. Structures which are not already gravitationally bound will ultimately fly apart. The Earth and the Milky Way would remain undisturbed while the rest of the universe appears to run away from us.

The nature of dark energy is currently a matter of speculation. Some believe that dark energy might be "vacuum energy", represented by the "cosmological constant" $(\Lambda)$ in general relativity, a constant uniform density of dark energy throughout all of space that is independent of time or the universe's expansion. This notion was introduced by Einstein, and is consistent with our limited observations to date. Alternatively, dark energy might vary with cosmic time. Only new kinds of observations can settle the issue.

\section{DARK MATTER AND ITS EVIDENCES}

Galaxies in our universe are rotating with such a speed that the gravity generated by their observable matter could not hold them together. Scientists think that there must be something which we cannot detect directly is giving these galaxies extra mass, generating the extra gravity they need for the purpose. This strange and unknown matter is termed as 'dark matter'. It can only be detected from its gravitational effects. It does not interact with the electromagnetic force due to which it does not absorb, reflect or emit light, making it extremely hard to detect. The most widely accepted theory of dark matter is that it's composed of weakly interacting massive particles (WIMPs) that only interact with the rest of the universe via the weak force and gravity, making them extraordinarily difficult to detect signals.

Dark matter is crucial to the Big Bang model of cosmology as a component which corresponds directly to measurements of the parameters with Friedmann cosmology solutions to general relativity. The cosmic microwave background anisotropies correspond to a cosmology where much of the matter interacts with photons more weakly than the known forces that couple light interactions to baryonic matter. Similarly, a significant amount of non-baryonic, cold matter is necessary to explain the large scale structure of the universe.

Historically, three categories of dark matter had been postulated. The categories cold, warm, and hot refer to how far the particles could move due to random motions in the early universe, before they slowed down due to the expansion of the Universe this is called the "free streaming length". Primordial density fluctuations smaller than this free-streaming length get washed out as particles move from overdense to underdense regions, while fluctuations larger than the free-streaming length are unaffected; therefore this free-streaming length sets a minimum scale for structure formation.

Today, cold dark matter is the simplest explanation for most cosmological observations. "Cold" dark matter is dark matter composed of constituents with a free-streaming length much smaller than the ancestor of a galaxy-scale perturbation. This is currently the area of greatest interest for dark matter 
research, as hot dark matter does not seem to be viable for galaxy and galaxy cluster formation, and most particle candidates become non-relativistic at very early times, hence are classified as cold.

Warm dark matter refers to particles with a freestreaming length comparable to the size of a region which subsequently evolved into a dwarf galaxy. This leads to predictions which are very similar to cold dark matter on large scales, including the CMB, galaxy clustering and large galaxy rotation curves, but with less small-scale density perturbations. This reduces the predicted abundance of dwarf galaxies and may lead to lower density of dark matter in the central parts of large galaxies; some researchers consider this may be a better fit to observations.

Hot dark matter are particles that have a freestreaming length much larger than a proto-galaxy size. An example of hot dark matter is already known: the neutrino. Neutrinos were discovered quite separately from the search for dark matter, and long before it seriously began: they were first postulated in 1930, and first detected in 1956. Neutrinos have a very small mass: at least 100,000 times less massive than an electron. Other than gravity, neutrinos only interact with normal matter via the weak force making them very difficult to detect (the weak force only works over a small distance, thus a neutrino will only trigger a weak force event if it hits a nucleus directly head-on). This would classify them as Weakly Interacting Light Particles, or WILPs, as opposed to cold dark matter's theoretical candidates, the WIMPs

Much of the evidence for dark matter comes from the study of the motions of galaxies. Many of these appear to be fairly uniform, so by the virial theorem, the total kinetic energy should be half the total gravitational binding energy of the galaxies. Experimentally, however, the total kinetic energy is found to be much greater: in particular, assuming the gravitational mass is due to only the visible matter of the galaxy; stars far from the center of galaxies have much higher velocities than predicted by the virial theorem. Galactic rotation curves, which illustrate the velocity of rotation versus the distance from the galactic center, cannot be explained by only the visible matter. Assuming that the visible material makes up only a small part of the cluster is the most straightforward way of accounting for this. Galaxies show signs of being composed largely of a roughly spherically symmetric, centrally concentrated halo of dark matter with the visible matter concentrated in a disc at the center. Low surface brightness dwarf galaxies are important sources of information for studying dark matter, as they have an uncommonly low ratio of visible matter to dark matter, and have few bright stars at the center which would otherwise impair observations of the rotation curve of outlying stars.

Gravitational lensing observations of galaxy clusters allow direct estimates of the gravitational mass based on its effect on light from background galaxies, since large collections of matter (dark or otherwise) will gravitationally deflect light. In clusters such as Abell 1689, lensing observations confirm the presence of considerably more mass than is indicated by the clusters' light alone. In the Bullet Cluster, lensing observations show that much of the lensing mass is separated from the X-ray-emitting baryonic mass. In July 2012, lensing observations were used to identify a "filament" of dark matter between two clusters of galaxies, as cosmological simulations have predicted.

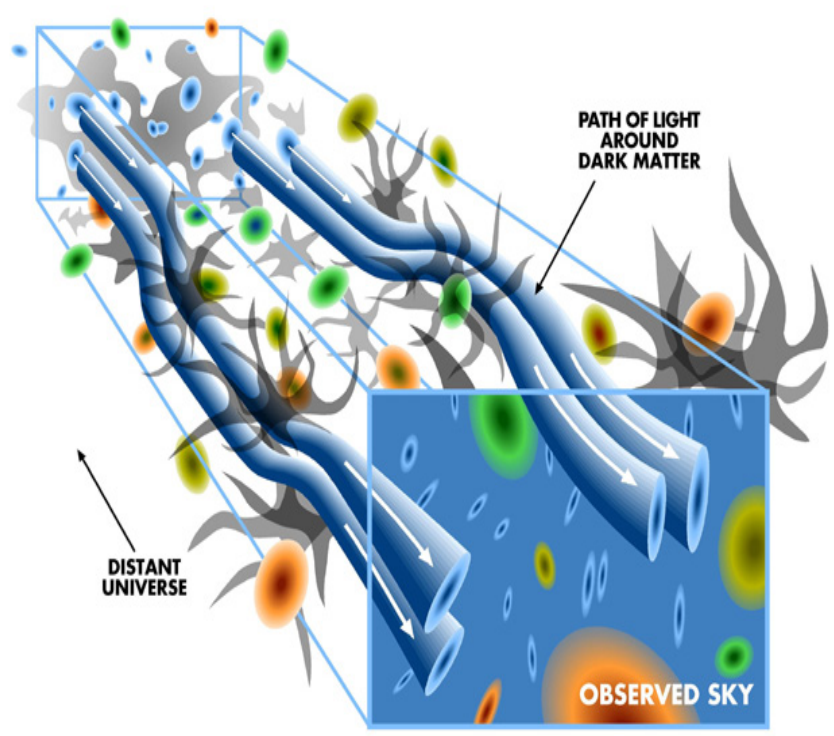

Figure: Path of light bending around dark matter 


\section{CONCLUSION:}

In the context of Einstein's general theory of relativity, one can formulate cosmological models, called Big Bang model. They give a reliable picture for the past fourteen billion years of the evolution of our universe which can be tested by comparing predictions and astronomical observations.

Remember that just because something cannot be seen doesn't mean it's not there. Dark matter is all around us in the universe, and there may even be dark matter passing through your body this second! We know that dark matter exists because we can observe its effects on other things in the universe; for example by studying how fast galaxies orbit around each other, we can estimate how much extra dark matter there must be in the system in addition to all of the visible matter we can see.

Despite successes, there are a number of open questions. The questions of dark matter, of dark energy, and last but not least the riddles of the very early universe should guarantee that cosmology remains a lively area of research for years to come

\section{REFERENCES}

[1] Alexandra witze and Nature News Blog; Scientific American, April 16, 2013

[2] Adam Riess, Encyclopedia Britannica, Dark energy (astronomy)

(www.britannica.com/EBchecked/topic/ darkenergy)

[3] Modern Cosmology: Max Camenzid

[4] Sears, Zeemansky, Young and Freedmann's University Physics, $12^{\text {th }}$ edition

[5] Stephan Hawking and Leonard Mlodinow: The Grand Design, 2011 Bantam Books.

[6] en.wikipedia.org/wiki/Dark_matter March 2013

[7] U. Seljak, Lectures on Dark Matter

[8] science.nasa.gov/astrophysics/focus-areas/ what-is-dark-energy/March 2013

[9] math.ucr.edu/home/baez/physics/Relativity/ GR/dark_matter, May 2013

[10] csep10.phys.utk.edu/astr162/lect/ cosmology/darkmatter, April 2013

[11] science.nationalgeographic.com/science/ space/dark-matter, April 2013

[12] hubblesite.org/hubble_discoveries/dark_ energy, May 2013

[13] www.smithsonianmag.com/.../DarkEnergy, May 2013

[14] www.lsst.org/lsst/public/darkenergy, April, 2013. 